\title{
3-Dimensional density profiles in edge plasma simulations for ICRF heating
}

\author{
Wouter Tierens ${ }^{1, \star}$, Jonathan Jacquot ${ }^{1}$, Wei Zhang $^{1,2}$, Jean-Marie Noterdaeme ${ }^{1,2}$, Volodymyr Bobkov ${ }^{1}$, Laurent \\ Colas $^{3}$, and The ASDEX Upgrade team ${ }^{1}$ \\ ${ }^{1}$ Max-Planck-Institut für Plasmaphysik, Boltzmannstrasse 2, D-85748 Garching, Germany \\ ${ }^{2}$ Ghent University, Sint-Pietersnieuwstraat 41, Gent, Belgium \\ ${ }^{3}$ CEA, IRFM, F-13108 Saint Paul-lez-Durance, France
}

\begin{abstract}
In this paper we discuss improvements made to two codes for the simulation of ICRF waves in edge plasmas: SSWICH-SW, which self-consistently models the interplay between sheath physics and radiofrequency waves (the slow wave), and RAPLICASOL, a Finite Element solver for Maxwell's equations in the cold plasma approximation. We have extended both to be able to handle 3D plasma density profiles. A comparison between a $1 \mathrm{D}$ and a 3D simulation reveals that the density profile dimensionality has a relatively small effect on $E_{\|}$at the aperture, but a large effect on the sheath potential at the antenna limiters
\end{abstract}

\section{Introduction}

It is important to understand the behaviour of Ion Cyclotron Range of Frequencies (ICRF) waves in edge plasmas and near the antenna, in order to understand the physical mechanisms underlying experimental observations such as ion sputtering [1, 2], RF induced convection [3], and variation in power coupling to the plasma.

Several codes are commonly used to numerically simulate these electromagnetic waves $[4,5]$. The vast majority, or perhaps all, of these codes until now use a 1D density profile, which depends only on the radial coordinate.

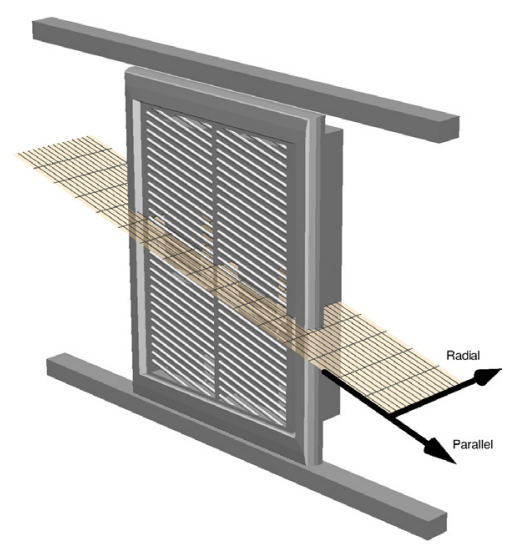

Figure 1. RAPLICASOL antenna geometry for the ASDEX Upgrade 2-strap antenna. A 2D radial-parallel slice used by the SSWICH-SW code is also shown. The tilt of this slice corresponds to the tilt of the background magnetic field: the SSWICHSW slice follows the field lines.

In this paper we discuss two codes in particular:
- RAPLICASOL (Radiofrequency wAve couPLing for Ion Cyclotron. Antenna in Scrape-Off-Layer [6]) to solve Maxwell's equations in cold plasma.

- SSWICH-SW (Self-consistent Sheaths and Waves for Ion Cyclotron Heating (Slow Wave) [7-9]) to selfconsistently determine the nonlinear plasma sheath potential and related quantities.

Both codes model physics in Cartesian coordinates, which neglect toroidal and poloidal curvature, but are nonetheless spatial coordinates, as opposed to codes such as TOPICA which operate in Fourier $\vec{k}$ space. Because the cold plasma dielectric tensor depends on quantities that depend on the spatial coordinates, it is relatively straightforward to extend them to handle 3D density profiles (or in fact 3D variation of other physical quantities such as the background magnetic field or the electron and ion temperature).

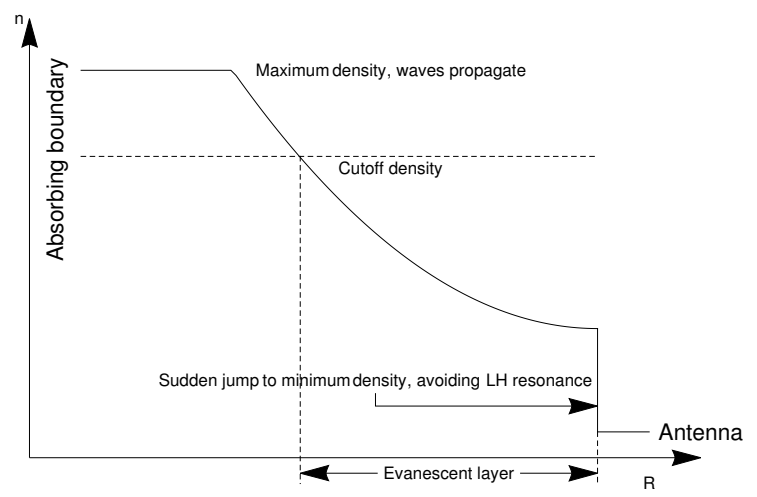

Figure 2. Summary of the density profile in RAPLICASOL.

\footnotetext{
^e-mail: wtt@ipp.mpg.de
} 


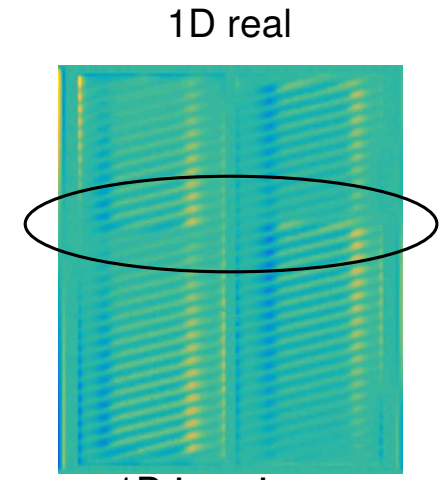

1D imaginary

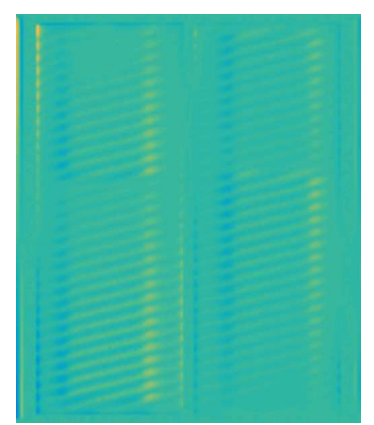

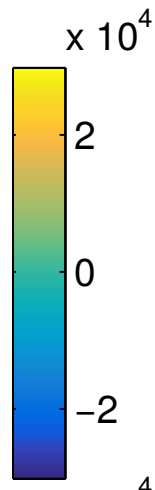
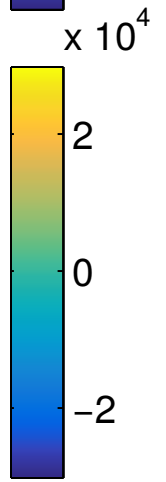

1D-3D difference (real)
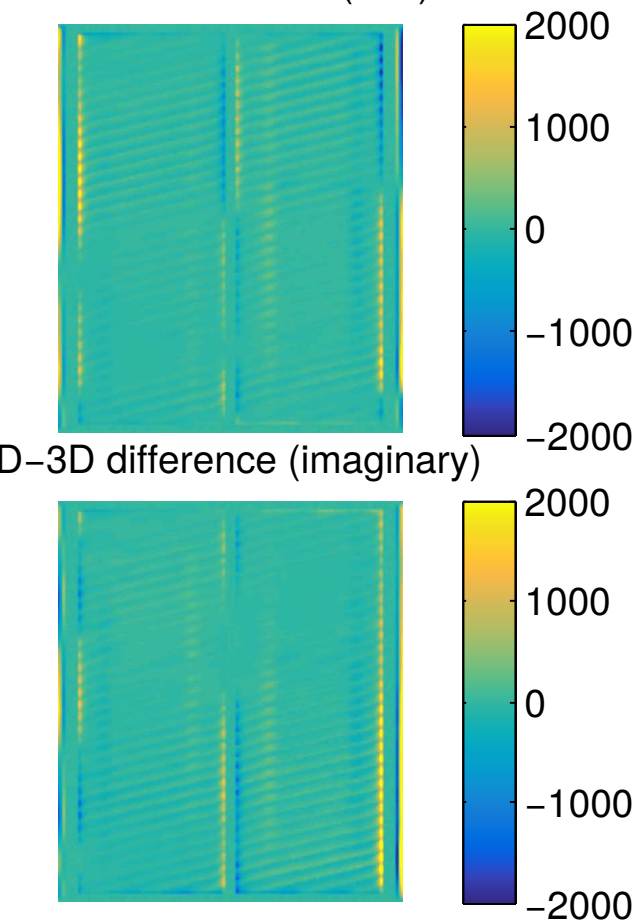

Figure 3. Comparison between $E_{\|}$field maps $(V / m)$ at the aperture of ASDEX Upgrade antenna 1 (a 2-strap antenna), calculated by RAPLICASOL with 1D resp. 3D density profiles. The left column contains $\mathfrak{R}\left(E_{\|, 1 D}\right)$ (top) and $\mathfrak{J}\left(E_{\|, 1 D}\right)$ (bottom). The right column contains the absolute difference between the field maps for the $1 \mathrm{D}$ and $3 \mathrm{D}$ density profiles: $\mathfrak{R}\left(E_{\|, 1 D}-E_{\|, 3 D}\right)$ and $\mathfrak{J}\left(E_{\|, 1 D}-E_{\|, 3 D}\right)$. The apparent horizontal discontinuity in $E_{\|, 1 D}$, indicated with an ellipse, is due to the way the straps are folded.

\section{RAPLICASOL}

RAPLICASOL is a 3D finite-element solver based on COMSOL, which solves Maxwell's equations in the cold plasma approximation in the neighbourhood of a realistic $3 \mathrm{D}$ antenna geometry as shown in figure 1 . RAPLICASOL does not model the core plasma: the simulation region is terminated by a layer of absorbing material (a so-called Perfectly Matched Layer or PML [11], adapted for cold magnetized plasma). By limitting the size of the simulation region, the PMLs also reduce the computational cost (RAM and CPU time) of the simulations.

There are two issues with allowing a completely arbitrary 3D density profile in RAPLICASOL. First, we should avoid the lower-hybrid resonance, which we do by imposing a discontinuous jump from vacuum to a certain minimum density (in SSWICH, we do not have to do this). Second, the density should be constant along the PML interface, which we ensure by imposing a maximum density. Although coupling and reflection depend on the radial gradient of the density, this maximum density does not have a large effect on the reflected waves, if it is chosen large enough for the waves to be propagating at that density (i.e. it should be larger than the cutoff density for all toroidal $k$ modes of interest). All this is summarized in figure 2.

\section{SSWICH-SW}

The SSWICH-SW code [7-9] self-consistently determines the nonlinear plasma sheath potential. Unlike RAPLICA-
SOL, it is essentially 2D: it operates on consecutive 2D radial-parallel slices, as shown in figure 1, and neglects derivatives in the vertical direction. SSWICH-SW imports an $E_{\|}$field map at the antenna aperture from RAPLICASOL, which excites the slow wave and the sheaths. SSWICH-SW solves three coupled fluid equations:

- The equation for the parallel electric field of the slow wave (SSWICH-SW models only the slow wave)

$$
\left(\epsilon_{\|} \Delta_{\|}+\epsilon_{\perp} \Delta_{\perp}+\epsilon_{\|} \epsilon_{\perp} \frac{\omega_{0}}{c^{2}}\right) E_{\|}=0
$$

where $\epsilon_{\|}, \epsilon_{\perp}$ are elements of the Stix tensor, $\Delta_{\|}, \Delta_{\perp}$ are the parallel resp. perpendicular terms in the laplacian, and $\omega_{0}$ is the antenna frequency. In SSWICH-SW's asymptotic mode [8], which we use in this paper, the boundary condition at the limiter surfaces is $E_{\|}=0$.

- The radiofrequency potential $V_{R F}$ at the limiters

$$
\epsilon_{\perp} \Delta_{\perp} V_{R F}= \pm \epsilon_{\|} \partial_{\|} E_{\|}
$$

- The DC current, given by a parallel and a perpencidular conductivity, is source-free in the bulk:

$$
\nabla \cdot J_{D C}=\left(\sigma_{\|} \Delta_{\|}+\sigma_{\perp} \Delta_{\perp}\right) V_{D C}=0
$$

The sheath boundary condition couples (2) and (3)

$$
\begin{aligned}
J_{D C \|} & =-\sigma_{\|} \nabla_{\|} V_{D C} \\
& =i^{+}\left(1-\exp \left(\frac{V_{f}+V_{b}-V_{D C}}{T_{e}}\right)\right)
\end{aligned}
$$



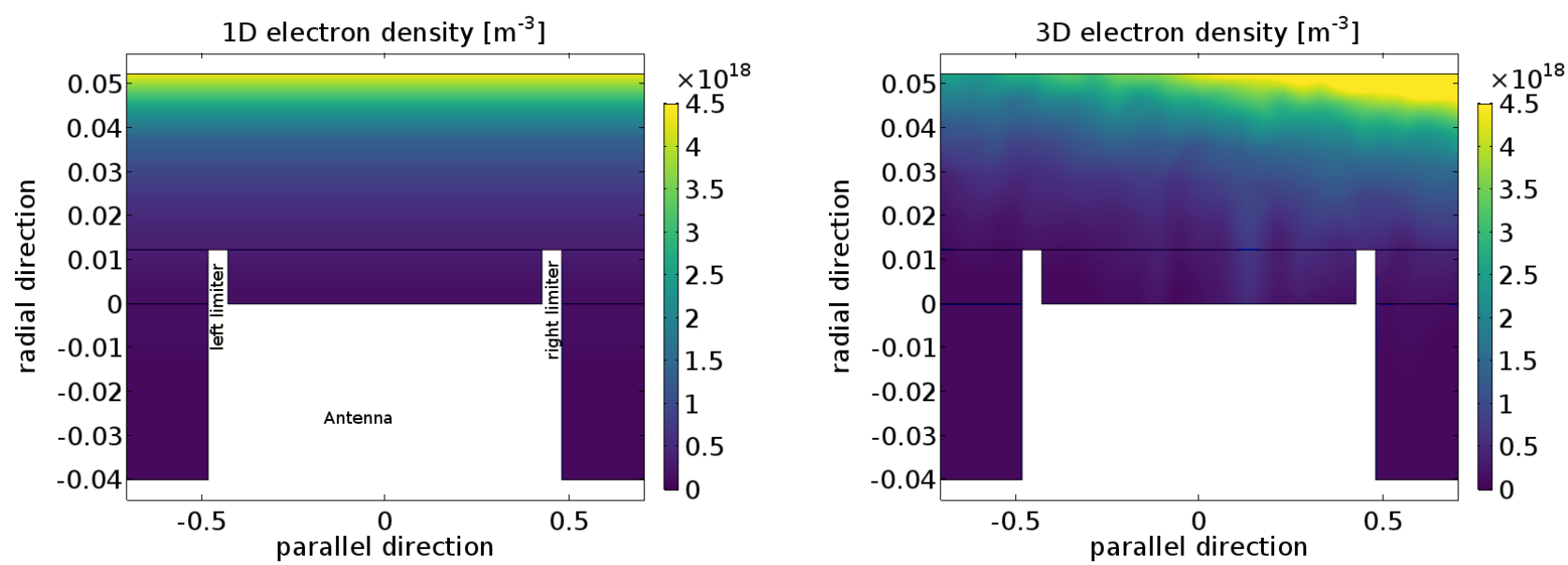

Figure 4. 1D and 3D electron density plotted along a radial-parallel SSWICH-SW slice at vertical position $0 \mathrm{~m}$. The density variation in the parallel direction is mostly due to poloidal, rather than toroidal, variation of the actual density profile, because the parallel direction is slanted.

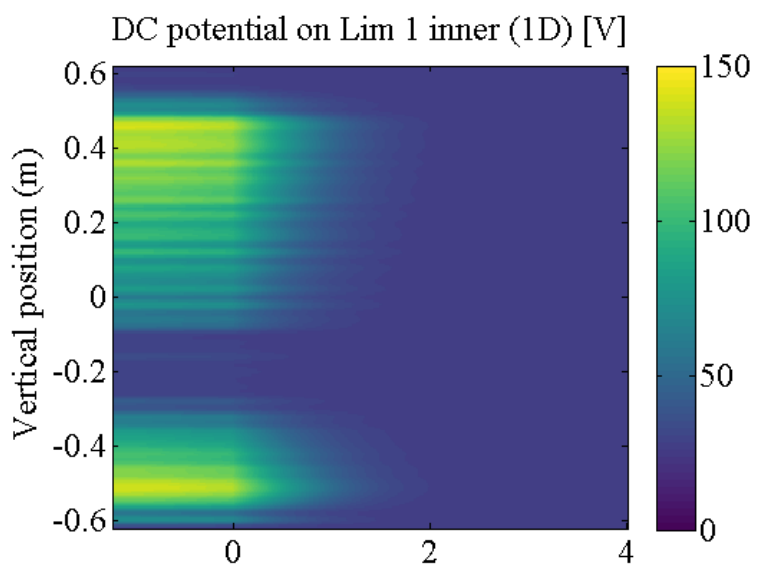

Distance from leading edge of limiter (cm)

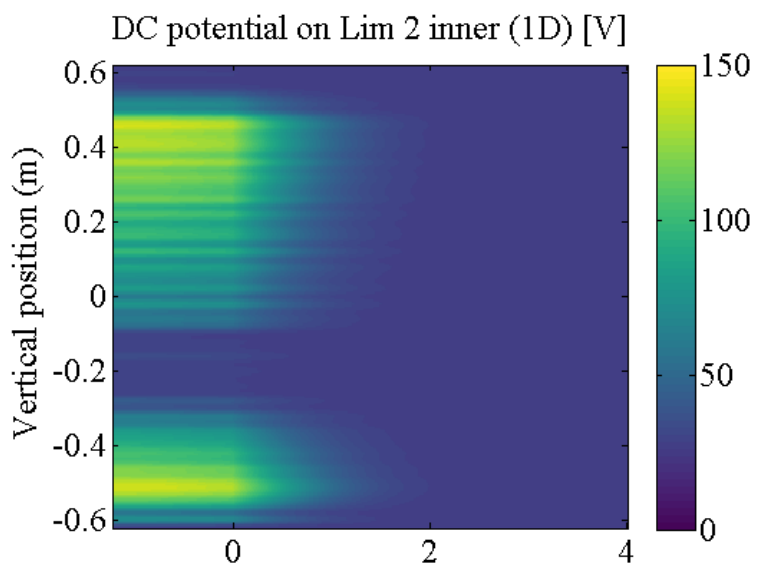

Distance from leading edge of limiter (cm)

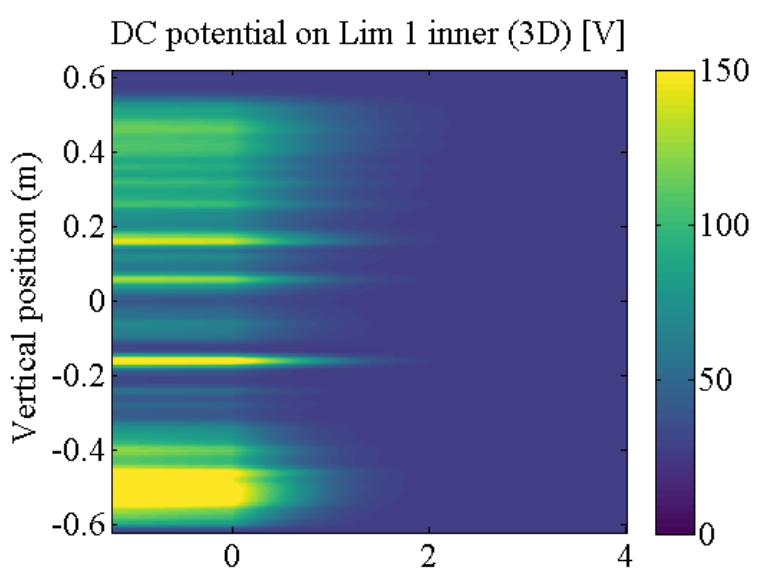

Distance from leading edge of limiter (cm)

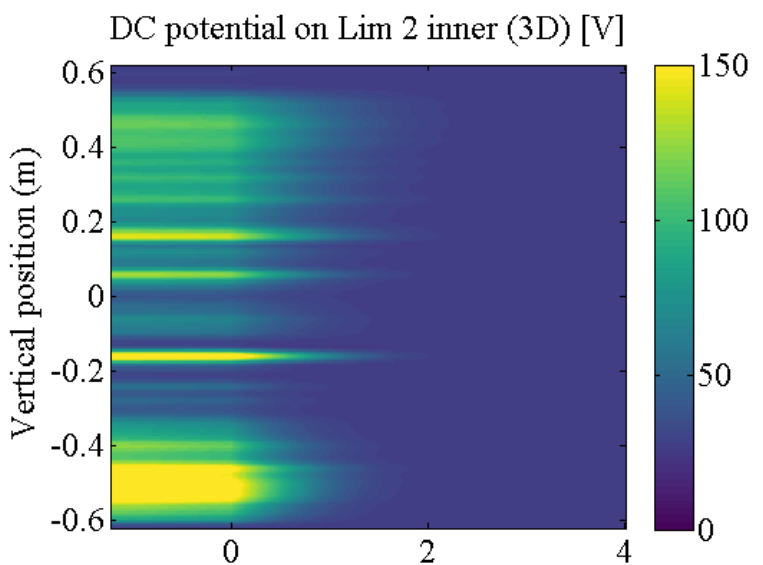

Distance from leading edge of limiter (cm)

Figure 5. DC potential on the inside of limiters 1 (left limiter) and 2 (right limiter) vs. radial and vertical coordinate, for 1D (left) and 3D (right) density profiles.

where $i^{+}$is the local ion saturation current, $V_{f}$ is the floating potential and $V_{b}$ is the biasing voltage which is a function of $V_{R F}$. In practice, (4) is limited by a saturation condition when the electron saturation current is reached. For further details see [7, 12]. 
These equations are density-dependent through the Stix tensor elements $\epsilon_{\|}$and $\epsilon_{\perp}$, but not through the DC conductivity $\sigma_{\|}$and $\sigma_{\perp}$, which are assumed to be proportional to the Spitzer conductivity (in reality, perpendicular DC currents are likely due to turbulence. The description in terms of a conductivity is purely phenomenological).

\section{Comparison between simulation results with 1D and 3D density}

In what follows we will use 3D density data determined using EMC3-Eirene for the "Mid-3" gas-puffing case in ASDEX Upgrade [13]. Let us call this $n_{\text {Mid3 }}(R, \theta, z)$. We will compare it with results obtained with the $1 \mathrm{D}$ density profile obtained by holding $\theta$ and $z$ constant: $n_{\text {Mid } 3}\left(R, \theta_{0}, z_{0}\right)$, where $\theta_{0}$ and $z_{0}$ are the coordinates of the center of the antenna.

In figure 3, we compare RAPLICASOL results with the $1 \mathrm{D}$ and $3 \mathrm{D}$ density profiles. The difference in $E_{\|}$field strength is the largest close to the right limiter, with a maximum difference in field strength of about $6 \mathrm{kV} / \mathrm{m}$. This difference in field strength is comparable to the typical field strength in either the $1 \mathrm{D}$ or the $3 \mathrm{D}$ case, although it is about an order of magnitude smaller than the maximum field strength. From the point of view of SSWICH-SW, which uses these field maps as input, the most important differences are those at or near the limiters [9]. The perport coupling resistance is calculated from the standing wave ratio at the ports with dipole phasing and $1 \mathrm{MW}$ of coupled power. In the $3 \mathrm{D}$ case it is $[2.08 \Omega, 2.20 \Omega]$ and in the $1 \mathrm{D}$ case $[2.14 \Omega, 2.18 \Omega]$, a difference of a few $\%$.

The SSWICH-SW code, as discussed before, operates on radial-parallel 2D slices (with the "parallel" direction being parallel to the background magnetic field). We have plotted the 1D and 3D density profile on one such slice in figure 4. Note that there is a poloidal variation as well: in addition to depending on the parallel coordinate as seen in figure 4 , the 3D density also changes from slice to slice.

SSWICH-SW calculates the nonlinear plasma DC potential along the limiters, and outputs $2 \mathrm{D}$ radial-vertical $V_{D C}$ maps on the inner and outer edge of each limiter (in SSWICH-SW, the limiter geometry is somewhat simplified: walls are either parallel or perpendicular to the magnetic field. This is necesary to satisfy the assumption that there is only slow wave propagation). It is these $V_{D C}$ maps which are shown figure 5. The short-wavelength vertical oscillations which appear even in the 1D case are due to the Faraday screen (the oscillations change when we change the number of Faraday screen bars, and disappear alltogether if there is no Faraday screen). The 3D density profile has a much larger effect on the DC potentials than on the $E_{\|}$field maps. The increase in $V_{D C}$ at the lower half of the antenna in the $3 \mathrm{D}$ case is likely due to the density which is lower there in the $3 \mathrm{D}$ density profile than in the 1D density profile.

\section{Conclusion}

We have extended the SSWICH-SW and RAPLICASOL codes to be able to handle 3D plasma density profiles. A comparison between results with 1D and 3D density profiles, where the 3D density profile was obtained using EMC3-Eirene and data from an ASDEX Upgrade gas puffing experiment, reveals that the change in density profile (at least for the specific parameters considered here) has a relatively small effect on the $E_{\|}$field map at the aperture and on the coupling resistance, but a large effect on the sheath potential at the antenna limiters.

\section{References}

[1] V. Bobkov, D. Aguiam, R. Bilato, S. Brezinsek, L. Colas, H. Faugel, H. Fünfgelder, A. Herrmann, J. Jacquot, A. Kallenbach et al., Plasma Physics and Controlled Fusion 59, 014022 (2016)

[2] V. Bobkov, M. Balden, R. Bilato, F. Braun, R. Dux, A. Herrmann, H. Faugel, H. Fünfgelder, L. Giannone, A. Kallenbach et al., Nuclear Fusion 53, 093018 (2013)

[3] W. Zhang, W. Tierens, J. Noterdaeme, V. Bobkov, D. Aguiam, D. Coster, H. Fünfgelder, J. Jacquot, R. Ochoukov, A. Silva et al., Submitted to Nuclear Fusion (2017)

[4] V. Lancellotti, D. Milanesio, R. Maggiora, G. Vecchi, V. Kyrytsya, Nuclear Fusion 46, S476 (2006)

[5] M. Brambilla, Plasma Physics and Controlled Fusion 35, A141 (1993)

[6] J. Jacquot, V. Bobkov, L. Colas, S. Heuraux, A. Křivská, L. Lu, J.M. Noterdaeme, R.I. Pinsker, Full wave propagation modelling in view to integrated ICRH wave coupling/RF sheaths modelling, in AIP Conference Proceedings (AIP Publishing, 2015), Vol. 1689, p. 050008

[7] J. Jacquot, Ph.D. thesis, Université de Lorraine (2013)

[8] L. Colas, J. Jacquot, S. Heuraux, E. Faudot, K. Crombé, V. Kyrytsya, J. Hillairet, M. Goniche, Physics of Plasmas 19, 092505 (2012)

[9] L. Colas, L. Lu, A. Křivská, J. Jacquot, J. Hillairet, W. Helou, M. Goniche, S. Heuraux, E. Faudot, Plasma Physics and Controlled Fusion 59, 025014 (2017)

[10] W. Zhang, V. Bobkov, J. Noterdaeme (2017), this conference

[11] J.P. Berenger, Journal of computational physics 114, 185 (1994)

[12] J. Jacquot, D. Milanesio, L. Colas, Y. Corre, M. Goniche, J. Gunn, S. Heuraux, M. Kubič, Physics of Plasmas 21, 061509 (2014)

[13] W. Zhang, V. Bobkov, J.M. Noterdaeme, W. Tierens, R. Bilato, D. Carralero, D. Coster, J. Jacquot, P. Jacquet, T. Lunt et al., Plasma Physics and Controlled Fusion 59, 075004 (2017) 